\title{
Unraveling resistive versus collisional contributions to relativistic electron beam stopping power in cold-solid and in warm-dense plasmas
}

\author{
B. Vauzour, ${ }^{1,2}$ A. Debayle, ${ }^{3,4}$ X. Vaisseau, ${ }^{1}$ S. Hulin, ${ }^{1}$ H.-P. Schlenvoigt, ${ }^{5}$ D. Batani, ${ }^{1,6}$ S.D. Baton, ${ }^{5}$ J.J. \\ Honrubia, ${ }^{3}$ Ph. Nicolaï, ${ }^{1}$ F.N. Beg, ${ }^{7}$ R. Benocci, ${ }^{6}$ S. Chawla, ${ }^{7}$ M. Coury, ${ }^{8}$ F. Dorchies, ${ }^{1}$ C. Fourment, ${ }^{1}$ E. \\ d'Humières, ${ }^{1}$ L.C. Jarrot, ${ }^{7}$ P. McKenna, ${ }^{8}$ Y.J. Rhee, ${ }^{9}$ V.T. Tikhonchuk, ${ }^{1}$ L. Volpe, ${ }^{6}$ V. Yahia, ${ }^{5}$ and J.J. Santos ${ }^{1, a)}$ \\ 1) Univ. Bordeaux, CNRS, CEA, CELIA (Centre Lasers Intenses et Applications), UMR 5107, F-33405 Talence, \\ France \\ ${ }^{2)}$ Laboratoire d'Optique Appliquée, ENSTA-CNRS-Ecole Polytechnique, UMR 7639, 91761 Palaiseau, \\ France \\ 3) ETSI Aeronáuticos, Universidad Politécnica de Madrid, Madrid, Spain \\ 4) CEA, DAM, DIF, F-91297 Arpajon, France \\ 5) LULI, Ecole Polytechnique CNRS/CEA/UPMC, 91128 Palaiseau Cedex, France \\ ${ }^{6)}$ Dipartimento di Fisica, Università di Milano-Bicocca, Milano 20126, Italy \\ 7) University of California, San Diego, La Jolla, California 92093, USA \\ ${ }^{8)}$ SUPA, Department of Physics, University of Strathclyde, Glasgow G4 ONG, \\ United Kingdom \\ ${ }^{9)}$ Korea Atomic Energy Research Institute (KAERI), Daejon 305-600, Korea
}

(Dated: 18 February 2014)

We present results on laser-driven relativistic electron beam propagation through aluminum samples, which are either solid and cold, or compressed and heated by laser-induced shock. A full numerical description of fast electron generation and transport is found to reproduce the experimental absolute $K_{\alpha}$ yield and spot size measurements for varying target thickness, and to sequentially quantify the collisional and resistive electron stopping powers. The results demonstrate that both stopping mechanisms are enhanced in compressed $\mathrm{Al}$ samples and are attributed to the increase in the medium density and resistivity, respectively. For the achieved time- and space-averaged electronic current density, $\left\langle j_{h}\right\rangle \sim 8 \times 10^{10} \mathrm{~A} / \mathrm{cm}^{2}$ in the samples, the collisional and resistive stopping powers in warm and compressed $\mathrm{Al}$ are estimated to be $1.5 \mathrm{keV} / \mu \mathrm{m}$ and $0.8 \mathrm{keV} / \mu \mathrm{m}$, respectively. By contrast, for cold and solid $\mathrm{Al}$, the corresponding estimated values are $1.1 \mathrm{keV} / \mu \mathrm{m}$ and $0.6 \mathrm{keV} / \mu \mathrm{m}$. Prospective numerical simulations involving higher $j_{h}$ show that the resistive stopping power can reach the same level as the collisional one. In addition to the effects of compression, the effect of the transient behavior of the resistivity of $\mathrm{Al}$ during $\mathrm{REB}$ transport becomes progressively more dominant, and for a significantly high current density, $j_{h} \sim 10^{12} \mathrm{~A} / \mathrm{cm}^{2}$, cancels the difference in the electron resistive stopping power (or the total stopping power in units of areal density) between solid and compressed samples. Analytical calculations extend the analysis up to $j_{h}=10^{14} \mathrm{~A} / \mathrm{cm}^{2}$ (representative of the full-scale fast ignition scenario of inertial confinement fusion), where a very rapid transition to the Spitzer resistivity regime saturates the resistive stopping power, averaged over the electron beam duration, to values of $\sim 1 \mathrm{keV} / \mu \mathrm{m}$.

PACS numbers: 52.50.-b,52.38.Kd, 52.65.-y, 52.70.La

\section{INTRODUCTION}

Fast Ignition $(\mathrm{FI})^{1}$ is an alternative approach to the conventional ignition scheme of Inertial Confinement Fusion (ICF) aiming at the development of an efficient energy production engine. In this two-step ignition scenario, a spherical pellet containing a Deuterium-Tritium (DT) mixture is adiabatically compressed by high intensity $\left(I \sim 10^{14-15} \mathrm{~W} . \mathrm{cm}^{-2}\right)$ long $(\sim \mathrm{ns})$ laser pulses with a total energy of the order ${ }^{2}$ of $\sim 250 \mathrm{~kJ}$. During DT stagnation, a relativistic electron beam (REB) is used to create an ignition spark that initiates the propagation of a thermonuclear burn in the compressed core. This REB is generated by a high intensity $\left(\sim 10^{20} \mathrm{~W} / \mathrm{cm}^{2}\right)$, high energy $(100 \mathrm{~kJ})$, short $(\sim 10 \mathrm{ps})$ laser pulse, close to

\footnotetext{
a)Electronic mail: santos.joao@celia.u-bordeaux1.fr
}

the critical density of the plasma corona surrounding the compressed core or at the tip of a cone embedded within the imploded capsule ${ }^{3}$. Due to the substantial reduction of the laser energy requirement compared to conventional ICF, higher gains are expected with a significant relaxation of the compression quality constraints.

At its source, the REB transports a total kinetic energy $\gtrsim 30 \%$ of the laser energy. A mean kinetic energy of 1 $2 \mathrm{MeV}$ is ideal to provide an efficient coupling to the core. For this, the REB has to propagate through a highly inhomogeneous plasma: from a low-density (few tenths of $\left.\mathrm{g} / \mathrm{cm}^{3}\right)$ plasma near the electron source, to a hightemperature $(\sim 300 \mathrm{eV})$ and high-density $\left(\sim 400 \mathrm{~g} / \mathrm{cm}^{3}\right)$ plasma close to the compressed core. In order to isochorically heat the compressed DT to thermonuclear conditions $(\sim 10 \mathrm{keV})$, the REB has to deliver an energy of $\sim 20 \mathrm{~kJ}$ in less than $20 \mathrm{ps}$ in a region of diameter less than $40 \mu \mathrm{m}^{4}$. Due to the large associated current density, $\sim 10^{14} \mathrm{~A} / \mathrm{cm}^{2}$, the REB transport is critically de- 
pendent on both collisional and collective mechanisms, such as important energy losses ${ }^{5-9}$, divergence ${ }^{10,11}$ and filamentation ${ }^{12-14}$, which could prevent the thermonuclear ignition. The success of FI therefore relies on understanding and characterizing such mechanisms. In particular, the collisional and resistive stopping powers should be accurately predicted, considering their important influence over the $\sim 100 \mu \mathrm{m}$ stand-off distance separating the REB source from the compressed core ${ }^{15}$.

Most experiments carried out so far exploring REB transport have involved cold and solid targets $7,9,10,16$. Only a few studies have explored the warm dense matter regime using laser-induced planar ${ }^{17-20}$ or cylindrical ${ }^{21,22}$ compression of the targets. In the case of a planar compression geometry, pioneering works ${ }^{17,18}$ were performed with a laser beam intensity not exceeding $10^{17} \mathrm{~W} / \mathrm{cm}^{2}$. However, this intensity regime is not relevant for FI, as intensities of $\sim 10^{19-20} \mathrm{~W} / \mathrm{cm}^{2}$ are required to generate a sufficiently energetic electron beam to trigger the fusion reactions. Although more recent experiments used laser pulses at the higher peak intensities ${ }^{19,20}$, an accurate quantification of the REB stopping power was not possible owing to the tracer layers signal enhancement by electron refluxing. Concerning the cylindrical compression geometry, the compressed regions had a small radial extent with significant consequences on the REB guiding and ruling out the possibility of a precise stopping power characterization as a function of the target material only (density, temperature, resistivity, etc.).

For the experiment described here, as in references ${ }^{17-20}$, we adopt a planar compression geometry with the REB injection in the opposite direction to the compressing shock propagation. Unlike in references ${ }^{17,18}$, in which electron transport was explored in room-temperature insulators, we use a metal as the sample material: Aluminum samples of variable thickness, embedded in a multi-layer foil target structure, were shock-compressed by a factor of 2 . This produced temperatures, upon sample compression, of $2-3 \mathrm{eV}$, not far short of the material Fermi temperature $\left(T_{F} \sim 11 \mathrm{eV}\right)$. The changes in density and temperature, increased respectively by factors $\sim 2$ and $\sim 100$, cause an increase in the material resistivity by more than one order of magnitude ${ }^{23,24}$. As the target areal density crossed by the REB is invariant during planar compression, it is, in principle, possible to experimentally discriminate collisional from resistive contributions to the REB energy losses by systematically comparing results from compressed to solid samples of the same initial thickness. Although the material and plasma parameters explored in this study are still far from those typical of a real FI scenario, the comparison of REB transport in warm-dense versus cold-solid matter using relativistic laser intensities is new. In particular, it provides evidence of non-negligible collective effects on REB energy losses, which are highly dependent on the temperature of the background medium ${ }^{25}$. The experimental results presented, supported by numerical simulations, show that the total REB stopping power is higher in compressed materials due to an increase of both the density and the resistivity of the sample material. Detailed analytical estimates confirm this behavior.

The paper is organized as follows. Section II describes the experimental setup, providing details of the laser synchronization and the target hydrodynamics presented in Section III. The codes used to simulate the REB generation and transport in our experimental conditions are described in Section IV. The experimental results are presented in Section V, and systematically compared to results from the simulation codes. The benchmarked runs are used to estimate the REB energy losses and stopping power in Section VI-A, which are confirmed by the calculations of a rigid-beam analytical model in Section VI-B. The main conclusions of this work are summarized in Section VII.

\section{EXPERIMENTAL SETUP AND TARGET DESIGN}

The experiment was carried out at the PICO2000 facil- 1 ity, LULI laboratory (École Polytechnique, France), using a dual-laser beam configuration: a long pulse (LP) laser beam to drive the target compression and an intense short pulse (SP) laser beam to generate the REB inside the same target (see Fig. 1). The $5 \times 5 \mathrm{~mm}^{2}$ surface-area targets were made of aluminum $(\mathrm{Al})$ of varying thickness $(10,20,40$ or $60 \mu \mathrm{m})$, also called the propagation layer, embedded between different K-shell fluorescence tracer layers: a $5 \mu \mathrm{m}$ silver $(\mathrm{Ag})$ layer, located at the front side (SP side), used to characterize the REB source (i.e. close to its generation zone) and two successive $10 \mu \mathrm{m}$ layers of tin $(\mathrm{Sn})$ and copper $(\mathrm{Cu})$, placed at the rear side (LP side), to characterize the fraction of the REB transmitted through the $\mathrm{Al}$ sample. To improve the compression quality and also to prevent direct laser ablation/heating of the $\mathrm{Cu}$ tracer, a $15 \mu \mathrm{m}$ polypropylene (PP) layer was added on the rear side of the target. Finally, a $5 \mu \mathrm{m}$ $\mathrm{Al}$ layer, coating the front surface of the target, enabled the generation of the fast electrons without damaging the $\mathrm{Ag}$ tracer layer. The LP laser beam was focused on the rear side PP layer with an intensity of $3 \times 10^{13} \mathrm{~W} / \mathrm{cm}^{2}$ at $\lambda=0.53 \mu \mathrm{m}$. The pulse temporal profile was flat-top with a $4.5 \mathrm{~ns}$ duration in order to homogeneously drive the compression in the longitudinal direction. Coupling a Phase Zone Plate (PZP) with the focusing lens enabled the production of a flat-top focal spot spatial profile of $400 \mu \mathrm{m}$ diameter, thus creating a large and homogeneous compressed area around the REB propagation axis. The $\mathrm{SP}$ laser beam was focused on the $\mathrm{Al}$ front layer and delivered $35 \mathrm{~J}$ pulses of $1.5 \mathrm{ps}$ duration Full Width at Half Maximum (FWHM) at $\lambda=1.06 \mu \mathrm{m}$. The focal spot was Gaussian-shaped with a diameter of $10 \mu \mathrm{m}$ (FWHM) containing $25 \%$ of the total energy, producing a peak intensity of $3 \times 10^{19} \mathrm{~W} / \mathrm{cm}^{2}$. Due to Amplified Spontaneous Emission (ASE), each pulse was preceded, ap- 
proximately $1.1 \mathrm{~ns}$ before, by a pedestal of $10^{12} \mathrm{~W} / \mathrm{cm}^{2}$. This was previously characterized via a comparison of the pre-plasma density profile interferometric measurements at the targets front side with $2 \mathrm{D}$ radiative hydrodynamic simulations ${ }^{19}$.

In order to characterize the fast electron population, K-shell fluorescence radiation, emitted as the fast electron pass through the different tracer layers, were collected by several diagnostics surrounding the target. A $\mathrm{X}$-ray imaging device ${ }^{26}$, composed of a spherical quartz crystal $(21 \overline{3} 1)$ in Bragg incidence $\left(\theta_{\text {Bragg }}=88.9^{\circ}\right)$ and positioned at $210 \mathrm{~mm}$ from the rear side of the target, enabled imaging of the $\mathrm{Cu}-K_{\alpha}$ emission $(\sim 8 \mathrm{keV})$ onto a CCD camera located $\sim 2.2 \mathrm{~m}$ away $^{27}$. The magnification of the system was thus $\sim 10.5$, and the spatial resolution was $\Delta x \sim 20 \mu \mathrm{m}$. Two different X-ray spectrometers were used to investigate REB energy losses ${ }^{25}$. The first one was a Cauchois-type hard X-ray Transmission Crystal Spectrometer (TCS) ${ }^{28}$ using a curved quartz crystal $(2 d=0.6884 \mathrm{~nm})$ to measure the absolute Sn$(\sim 25 \mathrm{keV})$ and $\mathrm{Ag}-K_{\alpha}(\sim 22 \mathrm{keV})$ yields from the target front side. The second one, a HOPG $(2 d=0.6714 \mathrm{~nm})$ reflection flat crystal spectrometer ${ }^{29}$ located at the target rear side, was employed to measure the absolute $\mathrm{Cu}-K_{\alpha}$ yields $(\sim 8 \mathrm{keV})$. Finally, a Streaked Optical Pyrometry (SOP) diagnostic, using a streak camera coupled to a visible radiation imaging device, was used during LPonly laser shots to measure the shock breakout time at the front side of the target relative to the start of the LP irradiation at the rear side (see the setup in Fig. 1).

\section{LASER BEAMS SYNCHRONIZATION AND HYDRODYNAMIC CHARACTERIZATION OF TARGET COMPRESSION}

Figure

We introduced a time delay $\tau$ between the SP and LP laser beams. The delay was selected depending on the desired state of the target (i.e. solid or compressed) and secondly on the thickness of the propagation layer ( $\mathrm{Al}$ sample). Indeed, in the case of compressed targets, the REB has to be generated when the shock has almost completely compressed the Al sample without perturbing the $\mathrm{Ag}$ tracer. In order to determine the values of $\tau$ for each target, 2D radiative-hydrodynamic simulations of the target compression have been performed using the code $\mathrm{CHIC}^{30}$. These simulations were benchmarked by SOP measurements of shock chronometry using specific targets without the front $\mathrm{Al}$ and $\mathrm{Ag}$ layers. The signals have been obtained by imaging the front side of the target, radiating at the shock breakout, onto the slit of a S20 streak camera. An example measurement is shown in Fig. 2. The temporal trace of the LP laser beam, used as a reference, is plotted on the left panel. Note that due to the absence of the laser beam amplification stages (so that the streak camera does not saturate) the temporal trace is ramp-like. This is a top-hat-like profile at full amplification. This time reference is compared to the

\begin{tabular}{ccccc}
\hline Initial Al sample thickness $[\mu \mathrm{m}]$ & 10 & 20 & 40 & 60 \\
\hline Delay $\tau_{\text {comp }}(S P / L P)[\mathrm{ns}]$ & 2.5 & 3.1 & 4.4 & 5.7 \\
Effective thickness $L_{\text {comp }}^{\mathrm{Al}}[\mu \mathrm{m}]$ & 6.3 & 12.2 & 23.6 & 35.1 \\
Areal density $\rho L_{\text {comp }}^{\mathrm{Al}}\left[\mathrm{mg} / \mathrm{cm}^{2}\right]$ & 2.6 & 5.3 & 10.6 & 15.9 \\
\hline Delay $\tau_{\text {solid }}(S P / L P)[\mathrm{ns}]$ & 1.9 & 1.9 & 1.9 & 1.9 \\
Effective thickness $L_{\text {solid }}^{\mathrm{Al}}[\mu \mathrm{m}]$ & 10 & 20 & 40 & 60 \\
Areal density $\rho L_{\text {solid }}^{\mathrm{Al}}\left[\mathrm{mg} / \mathrm{cm}^{2}\right]$ & 2.7 & 5.4 & 11.8 & 16.2 \\
\hline \hline
\end{tabular}

TABLE I. Summary of the different used time delays $\tau$ between SP and LP laser beams in the cases of compressed (top) or solid (bottom) targets. The final (i.e. at the moment of the electron injection inside the target) thickness as well as the final areal density of the $\mathrm{Al}$ sample, computed by $\mathrm{CHIC}$ hydrodynamic simulations, are also shown.

SOP trace obtained in the case of a target with a $20 \mu \mathrm{m}$ Al sample irradiated at LP full power (right panel). The time needed for the shock to transit the target is estimated to be $3.1 \mathrm{~ns}$. The results are obtained with an accuracy of $0.1 \mathrm{~ns}$, evaluated from the standard deviation of the laser jitter. The shock velocity, inferred from these measurements, is estimated to be $14-15 \mu \mathrm{m} / \mathrm{ns}$.

On-axis density and temperature profiles, computed 3 by the hydrodynamic simulations, are presented in Fig. 3 for the case of a $60 \mu \mathrm{m} \mathrm{Al}$ sample target at times just before (i.e. $50 \mathrm{ps}$ ) the REB injection. Using a short delay, $\tau=1.85 \mathrm{~ns}$ (top panel), enables the injection of the REB just before the shock reaches the Al-sample. Therefore, the REB propagates through cold $\left(T_{e}=0.03 \mathrm{eV}\right)$ and solid Al $\left(\rho=\rho_{0}=2.7 \mathrm{~g} / \mathrm{cm}^{3}\right)$. By contrast, the use of a long delay, $\tau=5.65 \mathrm{~ns}$ for the $60 \mu \mathrm{m}$-Al sample target (bottom panel), enables the shock to almost reach the $\mathrm{Ag} / \mathrm{Al}$ interface, effectively compressing the whole Al sample. In this latter case, the REB crosses a homogeneous warm $\left(T_{e} \sim 2-3 \mathrm{eV}\right)$ and dense Al sample $\left(\rho \sim 2 \rho_{0}\right)$. Note that for both cases, the front sides of the target (i.e. the front $\mathrm{Al}$ and $\mathrm{Ag}$ layers) are in similar conditions: the $\mathrm{Al}$ front layer is partially ablated due to the SP beam ASE intensity pedestal and the Ag tracer layer is kept intact. As can be seen on the left figure (solid case), the LP beam is used to introduce similar conditions (density and temperature) to the compressed case inside the rear side $\mathrm{Sn}, \mathrm{Cu}$ and $\mathrm{PP}$ layers. More particularly, it enables the generation of a long PP plasma tail (few hundreds of microns) that partly screens the rear side electrostatic field as well as slows down and confines the fast electrons, preventing them recirculating inside the different K-shell tracers ${ }^{9,31}$. Therefore, the measured Kshell fluorescence signals are produced by only one REB transit, facilitating the quantification of the REB stopping power inside the Al samples.

By virtue of the target areal density conservation, the planar compression enables experimental investigation of the importance of the resistive energy losses on the REB propagation by unequivocally decorrelating them from the collisional energy losses. More precisely, as illustrated in Table I, the Al sample is compressed to approximately twice its initial density $\rho_{0}$ while its length is re-
Figure 3

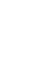


duced by the same factor. Consequently, its areal density $\rho L_{A l}$ along the REB propagation axis is maintained constant with the compression. As the electron collisional stopping power is to first approximation proportional to the density of the crossed medium, the collisional energy losses, integrated over the sample length, are approximately the same for a given initial sample thickness in both compressed and solid Al. The corollary fails regarding the resistive energy losses which should increase with the sample resistivity $\eta\left(\eta_{\text {solid }} \sim 3 \times 10^{-8} \Omega \mathrm{m} \rightarrow\right.$ $\eta_{\text {compressed }} \sim 5 \times 10^{-7} \Omega \mathrm{m}$, see Fig. 4 ) mainly due to the temperature raise from $0.03 \mathrm{eV}$ to $\sim 2-3 \mathrm{eV}$. At the value reached, close to the Fermi temperature $\left(T_{F} \sim 11 \mathrm{eV}\right)$, the resistivity is mostly governed by the electron-electron collisions and close to its maximum. For the full temperature range scanned during compression, below $T_{F}$, the resistivity depends on both density and temperature, respectively with negative and positive powers (see Section $\mathrm{IV}-\mathrm{B})^{23,24}$. However, it is the bigger change in temperature that determines the resistivity change between a Figure solid and a compressed sample.

\section{REB GENERATION AND TRANSPORT SIMULATIONS}

The simulation of both laser/plasma interaction and electron beam transport through dense matter involve different time and spatial scales, resulting in different numerical resolutions. While the laser/plasma interaction requires a fine description of the plasma skin depth (of several $\mathrm{nm}$ ), which can be obtained by resolving the kinetic Vlasov equations, the electronic transport demands a description of the solid background whether classical or quantical. Hence, the latter relies on a fluid description with appropriate equations of state of the background species. An alternative approach to describe the whole experiment is to use two different codes. A 2D cartesian Particle-in-Cell (PIC) simulation ${ }^{32}$ has been performed to calculate the electron distribution function produced by the SP interaction with the ASE-induced preplasma. Then, within some simplifications, the resulting electron function has been used as the initial beam condition in a $2 \mathrm{D}$ axisymmetric hybrid code of electron transport ${ }^{33}$.

\section{A. Particle-In-Cell simulations of the REB source}

The PIC simulation, set up to model the interaction of the SP beam on the front-Al-layer, assumes a similar electron density profile for all targets. The density profile, produced by the laser amplified spontaneous emission (ASE) pedestal, was measured by side-on interferometry in a previous experiment ${ }^{19}$ and successfully reproduced with the CHIC code (see Fig. 3). We thus use this profile as an initial condition in the PIC simulation. For numerical cost reasons, the density is limited to the maximum density $n_{e}=80 n_{c}$, where $n_{c}=m_{e} \varepsilon_{0} \omega_{0}^{2} / e^{2}$ is the critical density. The maximum density starts at $x=18 \mu \mathrm{m}$ and stretches as far as the end of the simulation box. The spatial and time resolutions are $\Delta x=\Delta y=c \Delta t=\lambda_{0} / 60$. Each cell contains 2 atoms of aluminum and 26 electrons. Collisions as well as ionizations are taken into account. The initial ionization level is self-consistently calculated using the Thomas-Fermi model. Absorbing conditions are applied to the fields, while escaping particles are reinjected with their initial temperature. The simulation box size is $80 \times 140 \mu \mathrm{m}^{2}$.

The p-polarized laser beam interacts in oblique inci- 5 dence $\left(\theta_{L}=45^{\circ}\right.$ with respect to the target surface normal). Its temporal profile is assumed to be Gaussian shaped with a peak intensity of $3 \times 10^{19} \mathrm{~W} / \mathrm{cm}^{2}$. To avoid any influence of the simulation box rear side ${ }^{34}$, the laser pulse duration is reduced to $0.5 \mathrm{ps}$ (full width at half maximum). In order to illustrate the absorption region and the electron beam ballistic, the magnetostatic field is plotted in Fig. 5 when the laser maximum intensity reaches the absorption region. The fast electrons are mainly generated around the laser/plasma boundary, that separates the strong magnetostatic field in the underdense plasma region from the Weibel induced magnetic fields in the overdense region ${ }^{35}$. The mechanism of acceleration, not yet fully understood, is probably a mixture of the Brunel heating ${ }^{36}$ and the $J \times B$ heating ${ }^{37-39}$. In the overdense plasma, the magnetic field filaments are attributed to a resistive filamentation instability ${ }^{12}$, with the characteristic scale length of the order of the beam skin depth. The REB source parameters, such as the angular and the kinetic energy distribution functions, are extracted at $21 \leq x \leq 22 \mu \mathrm{m}$, i.e. approximately $\sim 1.5 \mu \mathrm{m}$ beyond the absorption region, to ensure that the electron source is not perturbed by the evanescent waves and the Weibel induced magnetic fields.

The computed kinetic energy distribution is temporally averaged over the entire simulation time and fitted over the range $0.01<\varepsilon<20 \mathrm{MeV}$ by the following normalized function:

$$
f(\varepsilon)=\left\{\begin{array}{cl}
\left(\frac{E_{0}}{\varepsilon}\right)^{a} & \text { for } 0.01<\varepsilon<5 \mathrm{MeV} \\
N \exp \left(-\frac{\varepsilon}{T}\right) & \text { for } 5 \leq \varepsilon<20 \mathrm{MeV}
\end{array}\right.
$$

where $E_{0}=1.7 \mathrm{keV}, a=1.6, T=3 \mathrm{MeV}$ and $N=$ $1 \times 10^{-5}$ correspond to the fitting parameters. This function is plotted on Fig. 6 (red solid line) and compared to the one extracted from the particle-in-cell simulation (gray solid line). Note that particular attention is paid to accurately fit the low energy part of the spectrum, especially for electron energy ranging from $\sim 30 \mathrm{keV}$ to $\sim 5 \mathrm{MeV}$ where the Sn and $\mathrm{Ag} \mathrm{K}$-shell ionization cross sections reach local maxima [see, for example, the Sn Kshell ionization cross section on Fig. 6 (blue solid line with squares)]. As reported a few times in the literature ${ }^{40,41}$, this part of the spectrum is best described by a decreasing power-law function, defined by the parameter $a$, rather than the commonly used Maxwellian functions (represented by the red dashed lines). This precaution en- 
abled us to accurately reproduce the experimental absolute $K_{\alpha}$ yields as it will be demonstrated below. As a result, the electron mean kinetic energy is estimated to be $\sim 190 \mathrm{keV}$, a value much smaller than that given by Figure ponderomotive scaling $(\sim 0.5-1 \mathrm{MeV})$.

6 The mean angular dispersion of the electron source is fitted by the following angular distribution function ${ }^{11}$ :

$$
f(\theta, r)=\exp \left[\frac{-\left(\theta-\theta_{r}\right)^{2}}{\Delta \theta_{0}^{2}}\right],
$$

where $\theta_{r}=\arctan \left[\tan \left(30^{\circ}\right) y / y_{0}\right]+18^{\circ}$ is the mean radial angle with $y_{0}=20 \mu \mathrm{m}$ the initial REB radius, and $\Delta \theta_{0} \sim 55^{\circ}$ the dispersion angle ${ }^{42}$. As shown in Fig. 5 and measured in the fitting formula of $\theta_{r}$, the electron beam propagates with an angle of $\sim 18^{\circ}$ from the target normal direction. This relativistic electron propagation angle is a typical feature of oblique incident laser interaction with an overdense plasma. Indeed, the electron beam direction is, at the lowest order, defined by the well-known transverse momentum conservation of the electrons in the boosted frame ${ }^{43}$. As a result, the electron propagation angle $\alpha$ can be estimated by ${ }^{44} \sin \alpha=\sin \theta_{L} \sqrt{\frac{\gamma-1}{\gamma+1}}$. Hence, low energetic electrons would propagate in the direction normal to the target, while the ultra-relativistic electrons would propagate along the laser incident wave direction. Considering the mean electron energy of $190 \mathrm{keV}$ and $\theta_{L}=45^{\circ}$, the associated mean propagation angle would be of the order of $\alpha \sim 20^{\circ}$, a value consistent with the propagation angle measured in the simulation. Finally, the laser-to-fast electron conversion efficiency, calculated by temporally and spatially integrating the electron energy flux is estimated to be $30 \pm 10 \%$.

\section{B. Hybrid simulations of REB transport}

The kinetic and angular distribution functions as well as the laser-to-fast electron conversion efficiency computed above are used as input parameters for the 2D axisymmetric hybrid simulations of the REB transport using the code developed by J.J. Honrubia ${ }^{33}$. Given the small propagation angle value reported in the PIC simulation, the oblique beam propagation is neglected in the hybrid simulation. This simplification is justified since, in our case, this parameter doesn't change the simulation results much. The REB transport is computed through the entire target with either compressed or solid samples. The hybrid method neglects the high frequency effects, hence enabling a simplification of Maxwell equations by neglecting the Poisson equation and the displacement current in the Maxwell-Ampere equation. The fast electrons composing the injected beam, with the associated current density $j_{h}$, are modeled kinetically while the return current, $j_{e}$ formed by the background electrons, is described as an inertialess fluid ${ }^{12,33,45}$. The ini- tial density and temperature on-axis profiles of the different targets are extracted, at the REB injection time, from the hydrodynamic simulations (as in the examples presented above in Fig. 3, for the case of targets composed of a $60 \mu \mathrm{m} \mathrm{Al}$-sample). The total simulation time is set to $8 \mathrm{ps}$ with temporal and spatial resolutions of $1 \mathrm{fs}$ and $0.5 \mu \mathrm{m}$, respectively. Eight different cases, corresponding to the different initial thicknesses of the Al sample (10, 20,40 or $60 \mu \mathrm{m}$ ) and of its state (solid or compressed), are simulated. Almost $3 \times 10^{6}$ macro-particles are used to simulate the propagation of $\sim 4 \times 10^{14}$ electrons through the target. The collisions as well as the ionization processes are taken into account. The electrical resistivity of each material composing the target is computed using the classical Drude model: $\eta=m_{e} \nu / e^{2} n_{e}$, where $e$ and $m_{e}$ are the electron charge and rest mass, respectively, $n_{e}$ the background electron density and $\nu$ the harmonic mean of the electron collision frequency, given by the Eidmann-Chimier model ${ }^{23,24}$ :

$$
\nu^{-2}=\left(\nu_{e-p h}+\nu_{e-e}\right)^{-2}+\nu_{c}^{-2}+\nu_{s p}^{-2} .
$$

The terms $\nu_{e-p h}=2 g_{0} e^{2} T_{i} / 4 \pi \varepsilon_{0} v_{F} \hbar^{2}$ and $\nu_{e-e}=$ $g_{1} T_{e}^{2} / \hbar T_{F}$ refer to the electron-phonon and electronelectron collision frequencies, respectively. $T_{e}, T_{i}$ and $T_{F}=m_{e} v_{F}^{2} / 2$ are the electron, ion and Fermi temperature, respectively. $v_{F}=\hbar\left(3 \pi^{2} n_{e}\right)^{1 / 3} / m_{e}$ corresponds to the Fermi velocity. The parameter $g_{0}$ is calculated knowing the metal conductivity at the temperature $T_{i}$. The parameter $g_{1} \sim 1-10$ is approximately known for several metals. In the case of aluminum $g_{0}=1.25$ and $g_{1}=1$. The electron-electron collision frequency becomes important for an electron temperature higher than a decimal fraction of the Fermi temperature. The increase of the electron collision rate with the electron temperature is saturated above the Fermi temperature. Indeed, the mean time of flight between two collisions cannot be shorter than the time of flight over the interatomic distance $r_{0}=\left(3 / 4 \pi n_{i}\right)^{1 / 3}$. Consequently, the collision frequency has to be limited to the maximum rate $\nu_{c}=\left(k_{B} T_{e} / m_{e}\right)^{1 / 2} / r_{0}$. For electron temperature well above the Fermi temperature, the electron-ion collisions in a classical non-degenerated plasma become the dominant effect and the resistivity is given by the Spitzer formula: $\nu_{s p}=g_{2} Z^{*} e^{4} n_{e} \ln \Lambda / m_{e}^{1 / 2} T_{e}^{3 / 2}$, with $g_{2}=1 / \sqrt{2 \pi}\left(8 \epsilon_{0}\right)^{2}$. $Z^{*}$ is the ionization state and $\ln \Lambda$ the Coulomb logarithm

The electrical resistivities of aluminum, used in the simulations for the solid (blue dashed line) and compressed (red solid line) targets, are displayed on Fig. 4. As discussed above, the compression of the Al sample causes its resistivity to increase by a factor $\sim 17$.

The $K_{\alpha}$ radiation, generated by the different tracer layers $(\mathrm{Ag}, \mathrm{Sn}$ and $\mathrm{Cu}$ ), is calculated using the K-shell ionization cross section formula introduced by Hombourger ${ }^{46}$ as well as the semi-empirical formula for the $K_{\alpha}$ relaxation probability introduced by 
Bambynek et al. ${ }^{47}$. It enables reproduction of the $K_{\alpha}$ signal yields and size for comparison with the experimental data.

Two probes are set on the $\mathrm{Al}$ sample boundaries (i.e. at the $\mathrm{Ag} / \mathrm{Al}$ and $\mathrm{Al} / \mathrm{Sn}$ interfaces) in order to evaluate the resistive and collisional energy losses of the REB, integrated over the samples thickness.

\section{COMPARISON OF THE EXPERIMENTAL RESULTS WITH SIMULATIONS}

The experimental results are systematically compared to the hydrid transport simulations via the $K_{\alpha}$ emission, assuming a $40 \%$ laser-to-fast electron conversion efficiency. This value best reproduces the experimental absolute $K_{\alpha}$ yields. A first comparison concerns the $\mathrm{Cu}-K_{\alpha}$ spot size measured with the $2 \mathrm{D} \mathrm{Cu}-K_{\alpha}$ imager. Typical images obtained during the experiment are presented on Fig. 7 (left) for the solid and compressed cases. By measuring the mean diameter of the each $\mathrm{Cu}-K \alpha$ spots (averaged FWHM over the two main axis of the spot) it has been possible to infer the REB shape and its radial spreading evolution for growing sample effective thickness $L_{A l}$ (i.e. the real sample length crossed by the REB). The results, deconvoluted from the imagery system response (i.e. the spatial resolution), are plotted as a function of the effective thickness of the $\mathrm{Al}$ sample (Fig. 7; see also Table I for values of $L_{A l}$ ) and compared to the simulations. Good agreement is found between the simulations (open symbols) and the experimental data (full symbols). The REB divergence is estimated to $22 \pm 6^{\circ}$ (half-angle) in concordance with previous results obtained on solid targets with an equivalent laser beam intensity ${ }^{26,48}$. Furthermore, the increase of the $\mathrm{Al}$ temperature and density seems to not influence the REB divergence, which remains roughly the same for solid and compressed targets for our interaction conditions. The REB divergence appears to essentially depend on the Figure laser-matter interaction and generation processes ${ }^{35}$.

$7 \quad$ The absolute yields measured with the TCS (Sn- and $\left.\mathrm{Ag}-K_{\alpha}\right)$ and HOPG $\left(\mathrm{Cu}-K_{\alpha}\right)$ x-ray spectrometers are plotted in Fig. 8 as a function of the final thickness of the $\mathrm{Al}$ sample and compared to the simulation results. These yields are obtained by temporally and spatially integrating the $K_{\alpha}$ emission from the fluorescent tracer layers. Fairly good agreement is found between experimental (full symbols) and numerical (empty symbols) results especially for the Sn- and $\mathrm{Ag}-K_{\alpha}$ yields. Nonetheless, the number of measured $K_{\alpha}$ photons is not well reproduced numerically in the case of $\mathrm{Cu}-K_{\alpha}$ emission, although the general trend is. The factor $\sim 3$ disagreement could partly result from the observed anomalous broadening of the emission lines, as it can be seen on the example spectrum shown in Fig. 1. This behavior hasn't been fully explained but it could be due to aging or deterioration of the crystal. Concerning the data trends, we first observe that the $\mathrm{Ag}-K_{\alpha}$, a diagnostic of the REB source, remains roughly constant with the Al sample effective thickness $L_{A l}$. This indicates only small shot-toshot variations of the REB source even if a small difference can be noticed experimentally between the solid and compressed targets. The $\mathrm{Cu}$ - and $\mathrm{Sn}-K_{\alpha}$ yields, linked to the REB population that has crossed the Al sample, are both decreasing with the $\mathrm{Al}$ sample effective thickness. This is the signature of a progressive energy loss of the REB as it propagates deeper inside the target. Besides, the same results show that this behavior is even more pronounced for compressed targets, reflecting a more important slowing down of the REB in this case.

The $\mathrm{Sn}-K_{\alpha}: \mathrm{Ag}-K_{\alpha}$ ratio is plotted in Fig. 9. This ratio 8 removes any shot-to-shot variations of the REB source, especially the observed difference between compressed and solid targets (top right panel of Fig. 8). Besides, the $\mathrm{Sn}$ and $\mathrm{Ag}-K_{\alpha}$ yields considered here are measured with the same spectrometer (same crystal) and the respective photon energies, 25 and $22 \mathrm{keV}$, are also significantly close so that one can fairly assume the two tracers are sensitive to the same electron energies. Their ratio is therefore associated to the fraction of the REB population, with energies above $\sim 30 \mathrm{keV}$, that has crossed the $\mathrm{Al}$ sample. Moreover, because it is plotted as a function of the areal density $\rho L_{\mathrm{Al}}$, any observed differences between the solid and the compressed cases should be related to resistive energy losses (the collisional losses being the same, in both the compressed and solid $\mathrm{Al}$ samples, for a given areal density). The fact that the ratio decreases with increasing areal density confirms the results presented in Fig. 8. However, no striking difference can be found between the compressed and the solid data, indicating that in our interaction regime the resistive energy losses are possibly too weak, compared to collisional losses, to be experimentally observable. Furthermore, these results suggest that the higher decreasing rates as a function of $L_{A l}$ recorded for the compressed targets on the absolute Sn- and $\mathrm{Cu}-K_{\alpha}$ yields (bottom panels of Fig. 8), are mainly due to higher collisional effects associated with the sample density increased by the shock-compression.

Figure 9

\section{ESTIMATE OF THE REB ENERGY LOSSES AND STOPPING POWER}

\section{A. Simulations}

In summary, our experimental results, confirmed by Figure simulations, indicate that the REB energy deposition is higher in the case of compressed targets for a given $\mathrm{Al}$ sample thickness. The energy deposition seems to be mainly governed by collisional losses. The resistive energy losses, in turn, are either approximately the same in compressed and solid targets, and/or too weak to be experimentally observable. In order to understand these results, simulated $2 \mathrm{D}$ maps of the volumic density of collisional and resistive energy, deposited by the REB inside

Figure

\section{8}

8

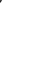


the targets, are plotted in Fig. 10 c), d), e) and f), for the case of $60 \mu \mathrm{m} \mathrm{Al}$ initial sample thickness. These maps correspond to the transverse section of the target, where the $x$ and $y$ axis represent the REB propagation axis and its associated radius, respectively. For every map, the continuous blue countour line represents the radius Half Width at Half Maximum (HWHM) for each $x$ position and the white dashed lines correspond to the Al sample boundaries. As illustrated in the panels c) and d), the highest collisional energy deposition over the Al-samples occurs in a zone of radius $\gtrsim 35 \mu \mathrm{m}$ at the left boundary, growing up to $\sim 65 \mu \mathrm{m}$ or to $\sim 90 \mu \mathrm{m}$ at the right boundaries of the compressed or solid samples respectively, even if multiple scattering events induce energy deposition over larger radii. The collisional energy deposition is clearly higher inside compressed targets due to the $\mathrm{Al}$ density increase $\left(\rho \approx 2 \rho_{0}\right)$. Unlike the collisional energy deposition, the resistive energy deposition [panels e) and f)] takes place over a narrower, quite well delimited region around the $\mathrm{REB}$ propagation axis $(y \lesssim 50 \mu \mathrm{m})$, corresponding to the region where the electron current density $j_{h}$ [panels a) and b)] is larger. For compressed targets, the resistive energy deposition occurs over larger radii due to the $\mathrm{Al}$ sample resistivity rise in the region under shock compression (see Fig. 4). Nonetheless, these additional resistive energy losses for the compressed targets remain very weak and thus not sufficient to be detectable experimentally. This is confirmed by the coherence, in terms of radial extent, between the plots of the final electronic temperature $T_{e}[\mathrm{~g})$ and $\mathrm{h}$ )] and the density of energy deposited by collisions [c) and d)], and by the fact that the values of $T_{e}$ achieved in both the compressed and solid $\mathrm{Al}$ samples as a function of Al-sample depth are roughly the same $\left(T_{e} \sim 100 \mathrm{eV}\right)$. Besides, the preponderant part of the energy losses occurs over the front $\mathrm{Al}$ and $\mathrm{Ag}$ layers in the first tens of microns, i.e. close to the SP laser interaction region, where the REB current density is maximum $\left(j_{h} \sim 10^{12} \mathrm{~A} / \mathrm{cm}^{2}\right)$. In this region, the resistive energy losses are even larger than the collisional losses as expected from other studies involving Figure solid Al targets ${ }^{7,49}$.

The role played by the current density $j_{h}$ on the REB losses is detailed in Fig. 11, where lineouts of deposited energy density (collisional and resistive) inside the $\mathrm{Al}$ sample are plotted as a function of the target depth for the compressed and solid cases. The lineouts are presented for various radii $r$ : $5 \mu \mathrm{m}, 20 \mu \mathrm{m}$ and $50 \mu \mathrm{m}$ where the mean current density $\left\langle j_{h}\right\rangle$, averaged temporally at the $\mathrm{Al}$ sample front surface and radially for $r \pm 5 \mu \mathrm{m}$, has been estimated to be $2.6 \times 10^{11} \mathrm{~A} / \mathrm{cm}^{2}, 1.6 \times 10^{11} \mathrm{~A} / \mathrm{cm}^{2}$ and $2.8 \times 10^{10} \mathrm{~A} / \mathrm{cm}^{2}$, respectively. At $r=5 \mu \mathrm{m}$, i.e. close to the REB propagation axis, the mean current density is high enough to result in the resistive energy deposition, proportional to $j_{h}^{2}$, being higher than the collisional one, proportional to $j_{h}$. Nonetheless, at such current density the resistive energy deposition seems not to depend on the sample state as this remains the same for both the compressed and solid targets. On the contrary, the col- lisional energy deposition is clearly higher in the case of compressed Al. At $r=20 \mu \mathrm{m}$, the resistive energy deposition decreases progressively as a function of depth, following the decrease of the mean current density from $\left\langle j_{h}\right\rangle=1.6 \times 10^{11} \mathrm{~A} / \mathrm{cm}^{2}$ at the sample front surface (i. e. at 0 on the plot) down to $\left\langle j_{h}\right\rangle=5.6 \times 10^{10} \mathrm{~A} / \mathrm{cm}^{2}$ at $30 \mu \mathrm{m}$ depth in the $\mathrm{Al}$ sample, and become less important than the collisional energy deposition: at $\sim 5 \mu \mathrm{m}$ for the compressed $\mathrm{Al}$ sample and $\sim 20 \mu \mathrm{m}$ for the solid one. The progressive decrease of $\left\langle j_{h}\right\rangle$ with increasing depth is accompanied by an increasing difference between the resistive energy losses in solid and compressed Al. These effects are even more pronounced for larger radii, as depicted in the right panel, where at $r=50 \mu \mathrm{m}$ the mean current density at the sample front surface is as low as $2.8 \times 10^{10} \mathrm{~A} / \mathrm{cm}^{2}$. The resistive energy deposition is now well below the collisional one and differences between the compressed and solid cases are clearly observable. It is evident that the value of $\left\langle j_{h}\right\rangle$ at the entrance of the Al sample can play a major role on the observed experimental differences in resistive losses between a solid and a compressed target. According to this, one could expect to clearly distinguish higher resistive losses for compressed targets when the mean current density $\left\langle j_{h}\right\rangle$ is between $\sim 10^{10} \mathrm{~A} / \mathrm{cm}^{2}$ and $\sim 10^{11} \mathrm{~A} / \mathrm{cm}^{2}$. For the present experimental conditions, $\left\langle j_{h}\right\rangle$ has been estimated to $\sim 8 \times 10^{10} \mathrm{~A} / \mathrm{cm}^{2}$ by temporally and spatially averaging the current density over a radius of $30 \mu \mathrm{m}$ around the REB propagation axis.

An estimate of the total REB energy losses is obtained by integrating the energy deposited by the two mechanisms over the whole Al sample. The results are presented in Fig. 12 (left panel) where the integrated resistive and collisional energy losses are plotted as a function of the final thickness of the $\mathrm{Al}$ sample for both the solid and compressed cases. The obtained values have been weighted by the energy of the laser beam in the present experiment $(35 \mathrm{~J})$. The collisional losses are clearly predominant in our laser interaction regime, while the resistive energy losses contribute to approximately one third of the total energy losses. In addition, these results suggest that both collisional and resistive energy losses are enhanced in compressed targets, as it has been observed above (Fig. 10 and Fig. 11), due to the growth of the density and the resistivity of the $\mathrm{Al}$ sample, respectively. The mean collisional and resistive stopping powers can now be estimated by averaging the energy losses over the REB duration, the REB spectrum and $35 \mu \mathrm{m}$ of crossed $\mathrm{Al}$ sample. The collisional stopping power, ranging from solid and cold to warm $(\sim 2 \mathrm{eV})$ and dense $\left(2 \rho_{0}\right) \mathrm{Al}$, is thus approximately $1.1-1.5 \mathrm{keV} / \mu \mathrm{m}$ and the resistive one $0.6-0.8 \mathrm{keV} / \mu \mathrm{m}$.

A growth of the mean REB current density can be explored through hybrid simulations, in particular to prospect changes to the resistive losses. A simple way consists in increasing the REB total energy, assuming an invariance on the fast electron distribution function and on the efficiency of the SP laser energy conversion
Figure 
into fast electrons. Full integrated 3D simulations including the effect of higher laser energy on the REB source (spectrum and total energy) are far beyond the scope of our objectives in this paper. The rise in the REB energy results in an increased number of fast electrons and therefore in a higher $j_{h}$, in virtue of the energy flux conservation equation between the laser pulse and the REB: the beam density is proportional to the laser intensity. With regard to this, we carried out simulations for two other SP laser beam energies : $E_{S P}=70$ and $350 \mathrm{~J}$. The results are presented in Fig. 12 (middle and right panels) where the integrated energy losses inside the Al sample have also been weighted by the corresponding energy of the SP laser beam. As illustrated in the different panels (and consistent with the mean electron-beam energy invariance), the rate of collisional loss is independent of the SP laser beam energy for both the solid and compressed cases. This behavior changes for the rate of resistive losses, which increases with the SP laser beam energy (and the electronic current density). Thus for $E_{S P}=70 \mathrm{~J}$, yielding $\left\langle j_{h}\right\rangle=1.5 \times 10^{11} \mathrm{~A} / \mathrm{cm}^{2}$ at the sample front surface, the resistive losses become comparable with the collisional losses inside $\mathrm{Al}$ over the first $20 \mu \mathrm{m}$ of propagation. Besides, the difference in resistive losses between the compressed and the solid cases tends to fade progressively as the laser energy becomes more important. The difference is almost nil when the energy of the laser increases to $E_{S P}=350 \mathrm{~J}$ and $\left\langle j_{h}\right\rangle$ reaches $10^{12} \mathrm{~A} / \mathrm{cm}^{2}$. For such high electronic current densities, the characteristic heating time up to the Fermi temperature, of the order of $\tau_{F} \sim 3 n_{e} T_{F} / 2 \eta j_{h}^{2} \approx 0.2 \mathrm{ps}$, is negligible compared to the REB duration. The medium is thus rapidly heated to temperatures where the resistivity, mainly governed by electron-ion collisions, decreases considerably according to $\eta \propto T_{e}^{-3 / 2}$ independently of the target density (Spitzer regime). Any initial difference in temperature, caused by the compression, is thus quickly erased. For such REB current density it would then not be possible, in the context of our experimental method, to discriminate between the resistive and collisional losses by comparing yields from solid and compressed targets, as they will no longer depend on the propagation medium density.

\section{B. Analytical estimates of resistive stopping power}

In this section we introduce a simple analytical formula which enables the resistive stopping power as a function of the REB current density $j_{h}$ and of the laser beam duration $\tau_{L}$ to be estimated. This formula is useful to predict the ranges for which the resistive stopping power is comparable or even higher than the collisional stopping power. In addition, it can predict the $\left(j_{h}, \tau_{L}\right)$-values for which the resistive stopping power, averaged over $\tau_{L}$, is significantly sensitive to the initial conditions, e.g. the initial temperature and density of the propagation medium.
The resistive stopping power, averaged over the beam duration $\tau_{L}$, can be reasonably approximated by the following expression:

$$
\left\langle\frac{\mathrm{d} \varepsilon}{\mathrm{d} x}\right\rangle_{t}=\frac{1}{\tau_{L}} \int_{0}^{\tau_{L}} e E \mathrm{dt}
$$

where $E$ corresponds to the electrostatic field generated by the electron beam. For a REB duration larger than the establishment time of the beam neutralization $(\sim$ few fs) and shorter than the neutralization duration (few tens of ps), the complete beam neutralization reduces to the Ohm's law ${ }^{50,51}: \mathbf{j}_{\mathbf{h}}=-\mathbf{j}_{\mathbf{e}}=-\mathbf{E} / \eta$, where $\mathbf{j}_{\mathbf{e}}$ represents the return current composed of the background thermal electrons. Therefore the plasma electron heating, described by the energy conservation equation, is given by:

$$
C_{e} \frac{\partial T_{e}}{\partial t}=\mathbf{j}_{\mathbf{e}} \cdot \mathbf{E}=\eta j_{h}^{2},
$$

where the ionization, the energy deposition by direct collisions and the heat conduction are neglected, and $C_{e}$ is the heat capacity of the plasma electrons at constant volume. It is worth noting that the ionization and the contribution of the direct collisions in the plasma heating are not negligible. However this simplification does not change the tendencies shown in this section.

Assuming that the REB current density is large enough $\left(j_{h}>10^{10} \mathrm{~A} / \mathrm{cm}^{2}\right)$ to heat the medium to a temperature far beyond the Fermi temperature $\left(T_{e} \gg T_{F}\right)$ it is possible to separate the collision frequency [Eq.(3)], and hence the resistivity, into four distinct regions, each one corresponding to a temperature range where a given collision process dominates, i.e. electron-phonon $\left(\nu_{e-p h}\right)$, electron-electron $\left(\nu_{e-e}\right)$ and electron-ion $\left(\nu_{s p}\right)$ collisions as well as the saturation regime $\left(\nu_{c}\right)$. Hence, the resistivity can be expressed as follows ${ }^{52}$ :

$$
\eta= \begin{cases}\eta_{e-p h} & \text { for } T_{0} \leq T_{e} \leq T_{1} \\ \eta_{1}\left(\frac{T_{e}}{T_{1}}\right)^{2} & \text { for } T_{1} \leq T_{e} \leq T_{2} \\ \eta_{2}\left(\frac{T_{e}}{T_{2}}\right)^{\frac{1}{2}} & \text { for } T_{2} \leq T_{e} \leq T_{3} \\ \eta_{3}\left(\frac{T_{e}}{T_{3}}\right)^{-\frac{3}{2}} & \text { for } T_{3} \leq T_{e}\end{cases}
$$

where

$$
\begin{aligned}
\eta_{e-p h} & =m_{e} \nu_{e-p h} / e^{2} n_{e} \\
\eta_{1} & =m_{e} \nu_{e-e}\left(T_{1}\right) / e^{2} n_{e} \\
\eta_{2} & =m_{e} \nu_{c}\left(T_{2}\right) / e^{2} n_{e} \\
\eta_{3} & =m_{e} \nu_{s p}\left(T_{3}\right) / e^{2} n_{e} .
\end{aligned}
$$

As the resistivity must be continuous, the transition temperatures can be deduced from the above equations: 


$$
\begin{aligned}
& T_{1}=\left(2 g_{0} e^{2} T_{i} T_{F} / 4 \pi \varepsilon_{0} g_{1} v_{F} \hbar\right)^{1 / 2} \quad \ll T_{F} \\
& T_{2}=\left(T_{F} \hbar / g_{1} m_{e}^{1 / 2} r_{0}\right)^{2 / 3} \sim T_{F} \\
& T_{3}=\left(g_{2} Z^{*} n_{e} e^{4} r_{0} \ln \Lambda\right)^{1 / 2} \gg T_{F}
\end{aligned}
$$

In the following calculation, we assume the Coulomb logarithm is equal to 4 .

In a similar way, the heat capacity is separated into two regions:

$$
C_{e}= \begin{cases}\frac{3}{2} n_{e} \frac{T_{e}}{T_{2}} & \text { for } T_{e} \leq T_{2} \\ \frac{3}{2} n_{e} & \text { for } T_{e}>T_{2}\end{cases}
$$

Within this simplification, for a degenerated state, $T_{e}<T_{2} \propto T_{F}$, the heat capacity is proportional to $n_{e} T_{e} / T_{F}$, consistent with a free fermion gas where $C_{e}=\pi^{2} T_{e} n_{e} /\left(2 T_{F}\right)$. For temperatures higher than $T_{2}$, the plasma electron gas can be considered as ideal and $C_{e}=3 n_{e} / 2$.

For an initial temperature $T_{0}$ smaller than $T_{1}$ (solid Al sample case) the integration of the energy conservation equation (5) provides the temporal evolution of the plasma electron temperature ${ }^{50}$ :

$$
T_{e}^{\text {solid }}(t)= \begin{cases}T_{0}\left(1+\frac{2 t}{\tau_{0}}\right)^{1 / 2} & \text { for } 0 \leq t \leq t_{1} \\ T_{1} \exp \left(\frac{t-t_{1}}{\tau_{1}}\right) & \text { for } t_{1} \leq t \leq t_{2} \\ T_{2}\left(1+\frac{t-t_{2}}{2 \tau_{2}}\right)^{2} & \text { for } t_{2} \leq t \leq t_{3} \\ T_{3}\left(1+\frac{5}{2} \frac{t-t_{3}}{\tau_{3}}\right)^{2 / 5} & \text { for } t \geq t_{3}\end{cases}
$$

where the characteristic heating times are:

$$
\begin{aligned}
\tau_{0} & =\frac{3 n_{e} T_{0}^{2}}{2 \eta_{e}-p h j_{h}^{2} T_{2}} \\
\tau_{1} & =\tau_{0} \frac{\eta_{e-p h}}{\eta_{1}} \frac{T_{1}^{2}}{T_{0}^{2}} \\
\tau_{2} & =\tau_{1} \frac{\eta_{1}}{\eta_{2}} \frac{T_{2}^{2}}{T_{1}^{2}} \\
\tau_{3} & =\tau_{2} \frac{\eta_{2}}{\eta_{3}} \frac{T_{3}}{T_{2}},
\end{aligned}
$$

and the transition times are:

$$
\begin{aligned}
& t_{1}=\left(T_{1}^{2} / T_{0}^{2}-1\right) \tau_{0} / 2 \\
& t_{2}=t_{1}+\tau_{1} \ln \left(T_{2} / T_{1}\right) \\
& t_{3}=t_{2}+2 \tau_{2}\left(\sqrt{T_{3} / T_{2}}-1\right) .
\end{aligned}
$$

Making use of Eqs. (5) and (4), the average electron stopping power can be rewritten as:

$$
\left\langle\frac{\mathrm{d} \varepsilon}{\mathrm{d} x}\right\rangle_{t}=\frac{e}{\left|j_{h}\right| \tau_{L}} \int_{T_{0}}^{T_{e f}} C_{e} \mathrm{dT}_{\mathrm{e}}
$$

where $T_{e f}$ is the final electron temperature, which is considered here to be $T_{e}\left(\tau_{L}\right)$. An integration of the previous relation, using Eq. (14), leads to the resistive electron stopping power:

$$
\left\langle\frac{\mathrm{d} \varepsilon}{\mathrm{d} x}\right\rangle_{t}=\left\{\begin{array}{ll}
\frac{3 e n_{e}}{2\left|j_{h}\right| \tau_{L}}\left(\frac{T_{2}}{2}-\frac{T_{0}^{2}}{2 T_{2}}+T_{e f}-T_{2}\right) & \text { for } T_{e f} \geq T_{2} \\
\frac{3 e n_{e}}{2\left|j_{h}\right| \tau_{L}}\left(\frac{T_{e f}^{2}}{2 T_{2}}-\frac{T_{0}^{2}}{2 T_{2}}\right) & \text { for } T_{e f}<T_{2}
\end{array},\right.
$$

where $T_{e f}=T_{e}\left(\tau_{L}\right)$ is obtained using Eq.(15).

In the case of a compressed target $\left(T_{0}>T_{1}\right)$ the REB doesn't experience the temperature domain where the $e$ $p h$ collisions occurs. Consequently, the equations must be modified in order to take this effect into account. The temporal evolution of the plasma electron temperature therefore reads:

$$
T_{e}^{c o m p}(t)= \begin{cases}T_{0} \exp \left(\frac{t}{\tau_{1}}\right) & \text { for } t \leq t_{2} \\ T_{2}\left(1+\frac{t-t_{2}^{\prime}}{2 \tau_{2}}\right)^{2} & \text { for } t_{2} \leq t \leq t_{3} \\ T_{3}\left(1+\frac{5}{2} \frac{t-t_{3}^{\prime}}{\tau_{3}}\right)^{2 / 5} & \text { for } t \geq t_{3}\end{cases}
$$

with $t_{2}^{\prime}=\tau_{1} \ln \left(T_{2} / T_{0}\right)$, and $t_{3}^{\prime}=t_{2}^{\prime}+2 \tau_{2}\left(\sqrt{T_{3} / T_{2}}-1\right)$, the other parameters remaining unchanged.

Calculations using equation (24) are presented in Fig. 13. The results are plotted for REB current density and duration $\left(j_{h}, \tau_{L}\right)$ for a range of conditions found in present short-pulse laser-plasma experiments $\left(j_{h} \lesssim 10^{12} \mathrm{~A} / \mathrm{cm}^{2}, \tau_{L} \sim 100 \mathrm{fs}\right)$ to the full-scale FI conditions $\left(j_{h} \lesssim 10^{14} \mathrm{~A} / \mathrm{cm}^{2}, \tau_{L} \sim 10 \mathrm{ps}\right)$, and of course including the parameters of the experiment described in this paper $\left(j_{h} \lesssim 10^{11} \mathrm{~A} / \mathrm{cm}^{2}, \tau_{L} \sim 1 \mathrm{ps}\right)$.

In the top and middle panels the results correspond- 13 ing to a cold and solid $\mathrm{Al}$ (i.e. $T_{0}=T_{i}=0.03 \mathrm{eV}$ and $\left.\rho=\rho_{0}=2.7 \mathrm{~g} / \mathrm{cm}^{3}\right)$ and to a warm and compressed $\mathrm{Al}$ (i.e. $T_{e}=T_{i}=2 \mathrm{eV}$ and $\rho=2 \rho_{0}=5.4 \mathrm{~g} / \mathrm{cm}^{3}$ ) are plotted, respectively. Both present a similar behavior, but with larger values in the case of a compressed target. Hence, for small current densities, such that the final temperature is smaller than the Fermi temperature, the averaged resistive stopping power is negligible. The strong increase of the resistive stopping power, associated with the strong electrical resistivity increase, occurs when the electron plasma becomes classical, $T_{e} \geq T_{F}$. For very high currents, such that the plasma resistivity enters the Spitzer regime, the averaged resistive stopping power decreases. Its maximum culminates at several $\mathrm{keV} / \mu \mathrm{m}$, for a well defined set of parameters $\left(j_{h}, \tau_{L}\right)$, where $T_{e f} \sim T_{F}$, i.e. the final resistivity corresponds to its maximum value (see Fig. 4).

We note that the resistive stopping power values obtained with this simple model for the parameters of the present experiment (indicated by the full blue circles in Fig. 13), of the order of $0.4 \mathrm{keV} / \mu \mathrm{m}$ for cold-solid and of $0.6 \mathrm{keV} / \mu \mathrm{m}$ for warm-compressed propagation media, are in good agreement with those estimated in Section VIA by comparing the $\mathrm{Sn}-K_{\alpha}: \mathrm{Ag}-K_{\alpha}$ ratio from both absolute yield data and simulation results: 0.5 and 
$0.8 \mathrm{keV} / \mu \mathrm{m}$, for solid and compressed Al-sample targets, respectively.

The ratio between the resistive electron stopping power in compressed and solid aluminum is presented in the bottom panel of Fig. 13, as a function of the beam current density and the beam duration. Again, two regions are well defined. For small current densities, the resistive stopping power is much higher for the compressed target while the difference tends to disappear for high current densities. The transition between the two regions coincides with the end of the electron quantum state in the case of the compressed target.

The aforementioned picture can be understood as follows. The electron temperature evolution, defined by Eq. (15), can be roughly simplified into two heating steps. Indeed, the heating time-scales $\tau_{2}$ and $\tau_{3}$, respectively associated with a plasma resistivity ruled by the electronion collisions in the saturation and Spitzer regimes, are negligible compared to $\tau_{1}$ dominated by the $e$ - $p h$ collisions. Hence, during the first heating step, the plasma is heated from the initial temperature, $T_{0}$, to the transition temperature $T_{1}$, in a time equal to $t_{1}$. Given the fast heating rate once the electron-electron collision appears, the plasma is instantaneously heated to the characteristic temperature $T_{3}$. For small laser beam duration, $\tau_{L} \leq t_{1}$, the resistive electron stopping power is entirely determined by the $e-p h$ collisions. Using Eqs. (15), and (24), it can be written as:

$$
\left\langle\frac{\mathrm{d} \varepsilon}{\mathrm{d} x}\right\rangle_{t}=e \eta_{e-p h} j_{h}=\frac{e m_{e}^{2} g_{0} T_{i} j_{h}}{2 \varepsilon_{0} 3^{\frac{1}{3}} \pi^{\frac{5}{3}} \hbar^{3} n_{e}^{\frac{4}{3}}} .
$$

This formula holds as long as the current density and beam duration verify the relation:

$$
j_{h}^{2} \tau_{L} \leq \frac{3 n_{e}}{4 \eta_{e-p h}}\left(\frac{T_{1}^{2}}{T_{2}}-\frac{T_{0}^{2}}{T_{2}}\right) .
$$

For higher beam current or longer beam duration, the resistive stopping power is determined by the Ohmic heating in the Spitzer regime, and is written as:

$$
\left\langle\frac{\mathrm{d} \varepsilon}{\mathrm{d} x}\right\rangle_{t}=\frac{3}{2}\left(\frac{5}{3}\right)^{\frac{2}{5}} e \eta_{3}^{\frac{2}{5}}\left(\frac{n_{e} T_{3}}{\tau_{L}}\right)^{\frac{3}{5}} j_{h}^{-\frac{1}{5}} .
$$

For small beam currents or short pulses, Eq. (26), the electron resistive stopping power increases with the ion temperature and decreases with the plasma electron density. In the previous example (Fig. 13), the (initial) temperature of the compressed target is higher by nearly two orders of magnitude than the (initial) temperature of the solid target, while the electron density is higher only by a factor of 2. The corresponding stopping power is thus increased by a factor of $\sim 25$ relative to the case of a coldsolid target. By contrast, the resistive stopping power in the Spitzer regime, Eq. (28), slowly increases with the electron plasma density $\left(\propto n_{e}^{3 / 5}\right)$, and decreases with the pulse duration and beam current. Hence, given the small compression factor, the resistive stopping power in the compressed target is just enhanced by $50 \%$.

For a small compression factor, the difference between solid and compressed targets is significant provided the final electron temperature is smaller or comparable to the Fermi temperature, i.e., provided the electron current density and pulse duration verify the inequality given in Eq. (27). For indication, the equality is plotted as a red dashed line in the top and middle panel of Fig. 13, for the case of the compressed target. The difference between resistive stopping power in compressed and in solid targets is noteworthy for parameters $\left(j_{h}, \tau_{L}\right)$ on the left of the dashed red line.

On the other hand, for the kind of plasma explored in our experiment, the resistive stopping power is comparable with the collisional stopping power provided that the former reaches $\left\langle\frac{\mathrm{d} \varepsilon}{\mathrm{d} x}\right\rangle_{t} \sim 1 \mathrm{keV} / \mu \mathrm{m}$. The isocontours of $\left\langle\frac{\mathrm{d} \varepsilon}{\mathrm{d} x}\right\rangle_{t}$, ranging from 0.5 to $7 \mathrm{keV} / \mu \mathrm{m}$, are presented in the same figure (top and middle panels): A small window exists such that the resistive stopping power is comparable with the collisional one, while being noticeably higher in the compressed case than in the solid case. For $\tau_{L}=1 \mathrm{ps}$ (common to $\mathrm{Nd}$ :Yag facilities) this window corresponds to $10^{11} \lesssim j_{h}<10^{12} \mathrm{~A} / \mathrm{cm}^{2}$, slightly above the regime explored in the present experiment.

In particular, the analytical estimation for the FI conditions $\left(j_{h}=10^{14} \mathrm{~A} / \mathrm{cm}^{2}, \tau_{L}=10 \mathrm{ps}\right)$ yields a time-averaged resistive stopping power not exceeding $\sim$ $1 \mathrm{keV} / \mu \mathrm{m}$. The calculation was made for a warm-dense metal, which in the FI scenario can be representative of the cone tip material heated by thermal or compression waves. However we have to recall that the ionization as well as collisional losses are not taken into account in this model. These simplifications could introduce a slight underestimation of the resistive stopping power, in particular when $j_{h}>10^{13} \mathrm{~A} / \mathrm{cm}^{2}$.

\section{CONCLUSIONS}

In conclusion, we performed an experiment in order to fully characterize the effects of background density and temperature rise, driven by laser-compression, on a relativistic regime of REB energy transport. Our experimental setup was designed to conserve the areal density of the $\mathrm{Al}$ propagation layer during the compression. In these specific conditions, the energy losses due to direct collisions of REB electrons with the background material can be separated from the energy losses due to the resistive effect. This difference was hardly visible in the experiment due to the weak but non-negligible contribution of the collective stopping power. To quantify the latter, we performed 3D REB-generation PIC simulations followed by $2 \mathrm{D}$ axis-symmetric REB-transport hybrid simulations, accounting for the complex collective effects such as the electrostatic field ionization, the collisional ionization and the three body recombination far from a local thermody- 
namic equilibrium. The simulations reproduced $\mathrm{K}_{\alpha}$ absolute yield and size measurements. We showed that the compression tends to increase the collisional and resistive REB stopping powers, due to the growth in density and resistivity, respectively. The effects of the transient behavior of $\mathrm{Al}$ resistivity during $\mathrm{REB}$ transport sum upon the effects of compression. Although the collisional losses are clearly the dominant process in our experimental interaction conditions $\left(\left\langle j_{h}\right\rangle \sim 8 \times 10^{10} \mathrm{~A} / \mathrm{cm}^{2}\right.$, at the Alsample front surface) the resistive processes are responsible for about one third of the total energy losses.

We underline that the benchmarked hybrid code and the analytical calculations both predict a range for the REB current density, $10^{11} \lesssim j_{h}<10^{12} \mathrm{~A} / \mathrm{cm}^{2}$ for pulse duration $\tau_{L}=1$ ps (slightly above our experimental conditions), where the resistive stopping power is comparable to the collisional one. Interestingly, a posterior similar experiment using the same geometry and targets, with a SP intensity above $10^{20} \mathrm{~W} / \mathrm{cm}^{2}$, has produced data consistent with our predictions: a measurable difference between compressed and solid data ${ }^{49}$. When $j_{h}$ approaches $10^{12} \mathrm{~A} / \mathrm{cm}^{2}$, the $\mathrm{Al}$ sample is rapidly $(\sim 0.2 \mathrm{ps}$, significantly smaller than $\tau_{L}$ ) heated to a temperature above the Fermi temperature where resistivity is governed by electron-ion collisions (Spitzer regime). Consequently, the material resistivity rapidly drops independently of its initial density/temperature (solid or compressed sample), saturating the time-averaged resistive stopping power. While not as accurate as the transport simulations, the analytical model helps to clearly understand the saturation mechanism. In particular, the analytical expansion of our investigation to the fast ignition conditions predicts that the resistive stopping power will not undermine the energy transport to the target dense core.

\section{ACKNOWLEDGMENTS}

The work presented here was performed under financial support from the Conseil Régional d'Aquitaine through project PETRA 20081304 005, and both the French National Agency for Research (ANR) and the competitiveness cluster Alpha - Route des Lasers through project TERRE ANR-2011-BS04-014. This study has been carried out in the frame of the Investments for the future Programme IdEx Bordeaux LAPHIA (ANR-10-IDEX03-02). The authors acknowledge LULI for giving access to the LULI2000 facility in the context of the preparatory phase of the HiPER project (Work Package 10: experimental validation program), as well as the support of the LULI2000 engineering staff in designing the setup implementation and during the experimental run. Alphanov is also gratefully acknowledged for the laser cutting of the targets. The numerical study was supported by Grant No. ENE2009-11668 of the Spanish Ministry of Education and Research and by the European Science Foundation SILMI program, and used HPC resources from
CeSViMa and from GENCI-CINES (Grants No. 2011056129 and No. 2012-056129). M.C. participated in this work thanks to funding from EPSRC (Grant number EJ/J003832/1). The authors gratefully acknowledge L. Gremillet for fruitful discussions.

${ }^{1}$ M. Tabak, J. Hammer, M. E. Glinsky, W. L. Kruer, S. C. Wilks, J. Woodworth, E. M. Campbell, M. D. Perry and R. J. Mason, Phys. Plasmas, 1, 1626 (1994)

${ }^{2}$ S. Atzeni, J. R. Davies, L. Hallo, J. J. Honrubia, P. H. Maire, M. Olazabal-Loum, J. L. Feugeas, X. Ribeyre, A. Schiavi, G. Schurtz, J. Breil and Ph. Nicolaï, Nucl. Fusion, 49, 055008 (2009)

${ }^{3}$ R. Kodama, P. A. Norreys, K. Mima, A. E. Dangor, R. G. Evans, H. Fujita, Y. Kitagawa, K. Krushelnick, T. Miyakoshi, N. Miyanaga, T. Norimatsu, S. J. Rose, T. Shozaki, K. Shigemori, A. Sunahara, M. Tampo, K. A. Tanaka, Y. Toyama, T. Yamanaka and M. Zepf, Nature, 412, 798 (2001)

${ }^{4}$ S. Atzeni, Phys. Plasmas, 6, 3316 (1999)

${ }^{5}$ V. T. Tikhonchuk, Phys. Plasmas, 9, 1416 (2002)

${ }^{6}$ A. J. Kemp, Y. Sentoku, V. Sotnikov, and S. C. Wilks, Phys. Rev. Lett., 97, 235001 (2006)

${ }^{7}$ J. J. Santos, A. Debayle, Ph. Nicolaï, V. Tikhonchuk, M. Manclossi, D. Batani, A. Guemnie-Tafo, J. Faure, V. Malka and J. J. Honrubia, Phys. Plasmas, 14, 103107 (2007)

${ }^{8}$ L. Volpe, D. Batani, A. Morace and J. J. Santos, Phys. Plasmas 20, 013104 (2013)

${ }^{9}$ L. Volpe, D. Batani, G. Birindelli, A. Morace, P. Carpeggiani, M. H. Xu, F. Liu, Y. Zhang, Z. Zhang, X. X. Lin, F. Liu, S. J. Wang, P. F. Zhu, L. M. Meng, Z. H. Wang, Y. T. Li, Z. M. Sheng, Z. Y. Wei, J. Zhang, J. J. Santos and C. Spindloe, Phys. Plasmas 20, 033105 (2013)

${ }^{10}$ J. S. Green, V. M. Ovchinnikov, R. G. Evans, K. U. Akli, H. Azechi, F. N. Beg, C. Bellei, R. R. Freeman, H. Habara, R. Heathcote, M. H. Key, J. A. King, K. L. Lancaster, N. C. Lopes, T. Ma, A. J. MacKinnon, K. Markey, A. McPhee, Z. Najmudin, P. Nilson, R. Onofrei, R. Stephens, K. Takeda, K. A. Tanaka, W. Theobald, T. Tanimoto, J. Waugh, L. Van Woerkom, N. C. Woolsey, M. Zepf, J. R. Davies, and P. A. Norreys, Phys. Rev. Lett., 100, 015003 (2008)

${ }^{11}$ A. Debayle, J. J. Honrubia, E. d'Humières, and V. T. Tikhonchuk , Phys. Rev. E, 82, 036405 (2010)

${ }^{12}$ L. Gremillet, G. Bonnaud and F. Amiranoff, Phys. Plasmas, 9, 941 (2002)

${ }^{13}$ M. Manclossi, J. J. Santos, D. Batani, J. Faure, A. Debayle, V. T. Tikhonchuk, and V. Malka, Phys. Rev. Lett. 96, 125002 (2006)

${ }^{14}$ J.J Honrubia and J. Meyer-ter-Vehn, Nuclear Fusion 46, L25 (2006)

${ }^{15}$ H. D. Shay, P. Amendt, D. Clark, D. Ho, M. Key, J. Koning, M. Marinak, D. Strozzi and M. Tabak, Phys. Plasmas 19, 092706 (2012)

${ }^{16}$ E. Martinolli, M. Koenig, S. D. Baton, J. J. Santos, F. Amiranoff, D. Batani, E. Perelli-Cippo, F. Scianitti, L. Gremillet, R. Mlizzi, A. Decoster, C. Rousseaux, T. A. Hall, M. H. Key, R. Snavely, A. J. MacKinnon, R. R. Freeman, J. A. King, R. Stephens, D. Neely, and R. J. Clarke, Phys. Rev. E, 73, 046402 (2006)

${ }^{17}$ T. A. Hall, S. Ellwi, D. Batani, A. Bernardinello, V. Masella, M. Koenig, A. Benuzzi, J. Krishnan, F. Pisani, A. Djaoui, P. Norreys, D. Neely, S. Rose, M. H. Key, and P. Fews, Phys. Rev. Lett. 81, 1003 (1998)

${ }^{18}$ D. Batani, J. R. Davies, A. Bernardinello, F. Pisani, M. Koenig, T. A. Hall, S. Ellwi, P. Norreys, S. Rose, A. Djaoui, and D. Neely, Phys. Rev. E 61, 5725 (2000)

${ }^{19}$ J. J. Santos, D. Batani, P. McKenna, S. D. Baton, F. Dorchies, A. Dubrouil, C. Fourment, S. Hulin, Ph. Nicolaï, M. Veltcheva, P. Carpeggiani, M. N. Quinn, E. Brambrink, M. Koenig, M. Rabec Le Glohaec, Ch. Spindloe and M. Tolley, Plasma Phys. Controll. Fusion, 51, 014005 (2009)

${ }^{20}$ J. J. Santos, D. Batani, P. McKenna, S. D. Baton, F. Dorchies, A. Dubrouil, C. Fourment, S. Hulin, E. d'Humières, Ph. Nicolä̈, L. Gremillet, A. Debayle, J. J. Honrubia, P. Carpeggiani, M. 
Veltcheva, M. N. Quinn, E. Brambrink and V. Tikhonchuk, J. Phys. Conf. Ser., 244, 022060 (2010)

${ }^{21}$ H. Nakamura, Y. Sentoku, T. Matsuoka, K. Kondo, M. Nakatsutsumi, T. Norimatsu, H. Shiraga, K. A. Tanaka, T. Yabuuchi, and R. Kodama, Phys. Rev. Lett. 100, 165001 (2008)

${ }^{22}$ F. Pérez, A. Debayle, J. Honrubia, M. Koenig, D. Batani, S. D. Baton, F. N. Beg, C. Benedetti, E. Brambrink, S. Chawla, F. Dorchies, C. Fourment, M. Galimberti, L. A. Gizzi, L. Gremillet, R. Heathcote, D. P. Higginson, S. Hulin, R. Jafer, P. Koester, L. Labate, K. L. Lancaster, A. J. MacKinnon, A. G. MacPhee, W. Nazarov, P. Nicolaï, J. Pasley, R. Ramis, M. Richetta, J. J. Santos, A. Sgattoni, C. Spindloe, B. Vauzour, T. Vinci, and L. Volpe, Phys. Rev. Lett., 107, 065004 (2011)

${ }^{23}$ K. Eidmann, J. Meyer-ter-Vehn, and T. Schlegel, Phys. Rev. E, 62, 1202, (2000)

${ }^{24}$ B. Chimier, V. T. Tikhonchuk, and L. Hallo, Phys. Rev. B, 75, 195124, (2007)

${ }^{25}$ B. Vauzour, J. J. Santos, A. Debayle, S. Hulin, H.-P. Schlenvoigt, X. Vaisseau, D. Batani, S. D. Baton, J. J. Honrubia, Ph. Nicolaï, F. N. Beg, R. Benocci, S. Chawla, M. Coury, F. Dorchies, C. Fourment, E. d'Humières, L. C. Jarrot, P. McKenna, Y. J. Rhee, V. T. Tikhonchuk, L. Volpe, and V. Yahia, Phys. Rev. Lett., 109, 255002 (2012)

${ }^{26}$ R. B. Stephens, R. A. Snavely, Y. Aglitskiy, F. Amiranoff, C. Andersen, D. Batani, S. D. Baton, T. Cowan, R. R. Freeman, T. Hall, S. P. Hatchett, J. M. Hill, M. H. Key, J. A. King, J. A. Koch, M. Koenig, A. J. MacKinnon, K. L. Lancaster, E. Martinolli, P. Norreys, E. Perelli-Cippo, M. Rabec Le Gloahec, C. Rousseaux, J. J. Santos, and F. Scianitti, Phys. Rev. E, 69, 066414 (2004)

${ }^{27}$ B. Vauzour, J.J. Santos, D. Batani, S.D. Baton, M. Koenig, Ph. Nicolaï, F. Perez, F.N. Beg, C. Benedetti, R. Benocci, E. Brambrink, S. Chawla, M. Coury, F. Dorchies, C. Fourment, M. Galimberti, L.A. Gizzi, R. Heathcote, D.P. Higginson, J.J. Honrubia, S. Hulin, R. Jafer, L.C. Jarrot, L. Labate, K. Lancaster, P. Köster, A.J. MacKinnon, P. McKenna, A.G. McPhee, W. Nazarov, J. Pasley, R. Ramis, Y. Rhee, C. Regan, X. Ribeyre, M. Richetta, F. Serres, H.-P. Schlenvoigt, G. Schurtz, A. Sgattoni, C. Spindloe, X. Vaisseau, L. Volpe, V. Yahia, Nucl. Inst. Meth. Phys. Res. A, 653, $176(2011)$

${ }^{28}$ J. F. Seely, L. T. Hudson, G. E. Holland, and A. Henins, Appl. Opt., 47, 2767 (2008)

${ }^{29}$ K. U. Akli, P. K. Patel, R. Van Maren, R. B. Stephens, M. H. Key, D. P. Higginson, B. Westover, C. D. Chen, A. J. Mackinnon, T. Bartal, F. N. Beg, S. Chawla, R. Fedosejevs, R. R. Freeman, D. S. Hey, G. E. Kemp, S. LePape, A. Link, T. Ma, A. G. MacPhee, H. S. McLean, Y. Ping, Y. Y. Tsui, L. D. Van Woerkom, M. S. Wei, T. Yabuuchi and S. Yuspeh, J. Inst. 5, P07008 (2010)

${ }^{30}$ P.-H. Maire, R. Abgrall, J. Breil, and J. Ovadia, SIAM J. Sci. Comput., 29, 1781 (2007)

${ }^{31}$ M N Quinn, X. H. Yuan, X. X. Lin, D. C. Carroll, O. Tresca, R. J. Gray, M. Coury, C. Li, Y. T. Li, C. M. Brenner, A. P. L. Robinson, D. Neely, B. Zielbauer, B. Aurand, J. Fils, T. Kuehl and P. McKenna, Plasma Phys. Controll. Fusion, 53, 025007 (2011)

${ }^{32}$ Y. Sentoku, A. J. Kemp, J. Comput. Phys., 227, 6846 (2008)

${ }^{33}$ J. J. Honrubia, M. Kaluza, J. Schreiber, G. D. Tsakiris and J. Meyer-ter-Vehn, Phys. Plasmas, 12, 052708 (2005)

${ }^{34}$ J. Tonge, J. May, W. B. Mori, F. Fiuza, S. F. Martins, R. A. Fonseca, L. O. Silva and C. Ren, Phys. Plasmas, 16, 056311 (2009)

${ }^{35}$ J.-C. Adam, A. Heron and G. Laval, Phys. Rev. Lett., 97, 205006 (2006)

${ }^{36}$ F. Brunel, Phys. Rev. Lett., 59, 52 (1987)

${ }^{37}$ W.L. Kruer and K. Estabrook, Phys. Fluids, 28, 430 (1985)

${ }^{38}$ J. Sanz, A. Debayle and K. Mima, Phys. Rev. E 85, 046411 (2012)

${ }^{39}$ A. Debayle, J. Sanz, L. Gremillet and K. Mima, Phys. Plasmas, 20, 053107 (2013)

${ }^{40}$ C. Ren, M. Tzoufras, F. S. Tsung, W. B. Mori, S. Amorini, R. A. Fonseca, L. O. Silva, J. C. Adam, and A. Heron, Phys. Rev.
Lett., 93, $185004(2004)$

${ }^{41}$ A. Debayle, J. J. Honrubia, E. d'Humières and V. T. Tikhonchuk, Plasma Phys. Control. Fusion, 52, 124024 (2010)

${ }^{42}$ A. Debayle, L. Gremillet, J. J. Honrubia and E. d'Humiéres, Phys. Plasmas, 20, 013109 (2013)

${ }^{43}$ A. Bourdier, Phys. Fluids, 26, 1804 (1983)

${ }^{44}$ M. Chen, Z.-M. Sheng and J. Zhang, Phys. Plasmas, 13, 014504 (2006)

${ }^{45}$ J. R. Davies, A. R. Bell, M. G. Haines, S. M. Guérin, Phys. Rev. E 56, 7193 (1997)

${ }^{46}$ C. Hombourger, J. Phys. B, 31, 3693 (1998)

${ }^{47}$ W. Bambynek, Proc. Conf. on X-ray and Inner Shell Processes in Atoms, Molecules and Solids, Aug. 2024, Leipzig, Germany (1984)

${ }^{48}$ J. J. Santos, F. Amiranoff, S. D. Baton, L. Gremillet, M. Koenig, E. Martinolli, M. Rabec Le Gloahec, C. Rousseaux, D. Batani, A. Bernardinello, G. Greison, and T. Hall, Phys. Rev. Lett. 89, $025001(2002)$

${ }^{49}$ J. J. Santos, D. Batani, S. D. Baton, F. N. Beg, T. Ceccotti, A. Debayle, F. Dorchies, J. -L. Feugeas, C. Fourment, L. Gremillet, J. J. Honrubia, S. Hulin, A. Morace, P. Nicolaï, F. P érez, H. Sawada, H. -P. Schlenvoigt, V. T. Tikhonchuk, X. Vaisseau, B. Vauzour, and M. Wei, J. Plasma Phys., 79, 4, 429 (2013)

${ }^{50}$ J.R. Davies, Phys. Rev. E 68, 056404 (2003)

${ }^{51}$ M. Passoni, V. T. Tikhonchuk, M. Lontano, and V. Yu. Bychenkov, Phys. Rev. E. 69, 026411 (2004)

${ }^{52}$ A. Debayle, Theoretical study of Ultra High Intensity laserproduced high-current relativistic electron beam transport through solid targets, PhD (2008) 
FIG. 1. (color online) Schematics of the experimental setup. The target design is detailed in the inset.

FIG. 2. (color online) Example of SOP measurements obtained by comparing the temporal trace of the LP laser beam (reference low energy flux shot without target; left panel) and the radiative emission following shock breakout at the front side of a $20 \mu \mathrm{m}$ $\mathrm{Al}$-sample target (without the front $\mathrm{Al}$ and $\mathrm{Ag}$ layers ) and using the LP laser at full power (right panel).

FIG. 3. (color online) On-axis density (red solid lines) and temperature (blue dashed lines) profiles obtained with 2D CHIC hydrodynamic simulations for the case of a $60 \mu \mathrm{m} \mathrm{Al} \mathrm{sample} \mathrm{target} \mathrm{either} \mathrm{solid} \mathrm{(top} \mathrm{panel)} \mathrm{or} \mathrm{compressed} \mathrm{(bottom} \mathrm{panel).}$ The dotted green line locates the shock front position. The red and green arrows represent the SP (i.e. REB) and LP (shock) directions, respectively. Their tips position indicate approximately the critical density surface for the SP laser and the shock front. The colored areas above the density profiles delimit the different material distribution in the target.

FIG. 4. (color online) Evolution of the aluminum electrical resistivity as a function of the background electron temperature $T_{e}$, for a solid (blue dashed line) or compressed (red solid line) target. Concerning the solid case, $T_{i}$ is kept constant and equal to $0.03 \mathrm{eV}$. In contrast, for the compressed case $T_{e}$ is set equal to $T_{i}$, meaning that we assume a thermodynamic equilibrium in the Al sample. The initial resistivities (prior to the REB injection) are pointed out by the open circles for the two target states. These two curves have been calculated following the Eidmann-Chimier model ${ }^{23,24}$ described in the next section.

FIG. 5. (color online) Magnetostatic field $e\left|B_{z}\right| / m_{e} \omega_{0}$ in logarithmic scale at the time when the maximum of the laser field reaches the absorption region. The white dashed line refers to the preplasma/target boundary.

FIG. 6. (color online) Simulated (PIC) REB distribution function (gray line) and its associated fits: the red solid line corresponds to the fit $f(\varepsilon)$ given by $(1)$, and the two red dashed lines to the often used sum of exponential decreasing fits. The blue solid line with squares represents the Sn K-shell ionization cross section.

FIG. 7. (color online) (left) Set of typical Cu- $K_{\alpha}$ images obtained during the experiment. (right) Evolution of the Cu- $K_{\alpha}$ spot size as a function of the effective thickness of the Al sample (see also Table.I).

FIG. 8. (color online) Evolution of the experimental (full symbols) and simulated (empty symbols) absolute Ag- $K_{\alpha}$ (top right), $\mathrm{Cu}-K_{\alpha}$ (bottom left) and $\mathrm{Sn}-K_{\alpha}$ (bottom right) yields as a function of the final thickness of the solid (blue) or compressed (red) Al sample.

FIG. 9. (color online) Comparison of the experimental and simulated evolution of the Sn- $K_{\alpha}:$ Ag- $K_{\alpha}$ ratio as a function of the $\mathrm{Al}$ sample areal density $\rho L_{\mathrm{Al}}$.

FIG. 10. (color online) 2D maps of the electronic current density at $t=1.5 \mathrm{ps}$ (top), the final volumic density of collisional and resistive energy deposited inside the target (middle), and the final electronic temperature (bottom) computed by the hybrid simulations. The results are presented for solid (left) and compressed (right) target composed initially of a $60 \mu \mathrm{m} \mathrm{Al} \mathrm{sample.}$ All the color values are in logarithmic scales. The white dashed lines correspond to the Al sample boundaries. The continuous blue countour lines represent the radius HWHM for each longitudinal position. The red arrows represent the SP laser position.

FIG. 11. (color online) Volumic density of energy deposited, by resistive (orange) or collisional dragging (green), inside the compressed (dashed line) or solid (solid line) Al sample as a function of target depth (relative to the REB injection surface) and for different radii $\langle r\rangle$ from the REB propagation axis: $5 \mu \mathrm{m}$ (left), $20 \mu \mathrm{m}$ (middle) and $50 \mu \mathrm{m}$ (right). The mean current densities labeling the plots correspond to the time-averaged and $r \pm 5 \mu \mathrm{m}$ averaged values at the sample front surface (corresponding to 0 on the plots).

FIG. 12. (color online) Estimates of collisional (green squares) and resistive (orange triangles) REB energy losses inside the Al sample as a function of its effective thickness (open and full symbols respectively for compressed and solid samples), assuming different SP laser beam energies: 35 (left panel: parameters of the present experiment), 70 (middle panel) and $350 \mathrm{~J}$ (right panel). For the three cases we assumed a 40\% energy conversion efficiency from the laser pulse to the REB. The energy loss values are normalized to the respective laser energy. 
FIG. 13. (color online) Resistive electron stopping power $[\mathrm{keV} / \mu \mathrm{m}]$ calculated according to Eqs. (15) and (24) against the current density $j_{h}$ and the beam duration $\tau_{L}$ for cold Al at solid density: $Z^{*}=3, \rho=2.7 \mathrm{~g} / \mathrm{cm}^{3}, T_{0}=T_{i}=0.03 \mathrm{eV}($ Top panel) and for compressed Al: $Z^{*}=3, \rho=5.4 \mathrm{~g} / \mathrm{cm}^{3}, T_{0}=T_{i}=2 \mathrm{eV}$ (Middle panel). The ratio of resisitive stopping power between compressed and solid $\mathrm{Al}$ is presented in the Bottom panel. The black lines represent the isocontours. The dashed red lines in the top and middle panels correspond to the equality of Eq. (27). The full blue circles indicate the parameters of the present experiment (i.e. $j_{h} \approx 8 \times 10^{10} \mathrm{~A} / \mathrm{cm}^{2}$ and $\tau_{L} \sim 1 \mathrm{ps}$ ). 


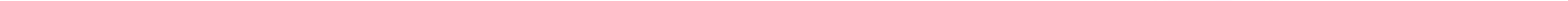


Thermal emission when the shock breaks out at the sample front side $t_{0}+3.1 \mathrm{~ns}$

Biginning of the laser pulse 
---- Temperature

Density

\section{Solid $\tau=1.85 \mathrm{~ns}$}

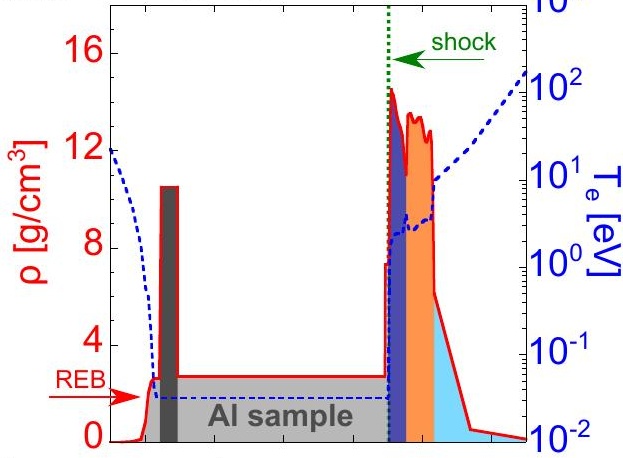

\section{Compressed $\tau=5.65 \mathrm{~ns}$}

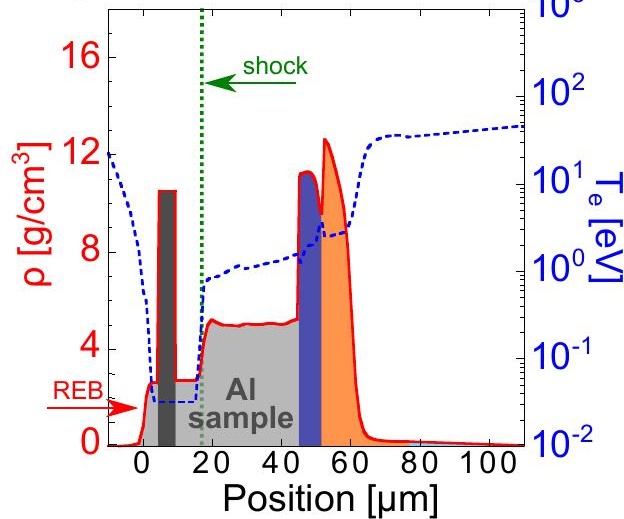




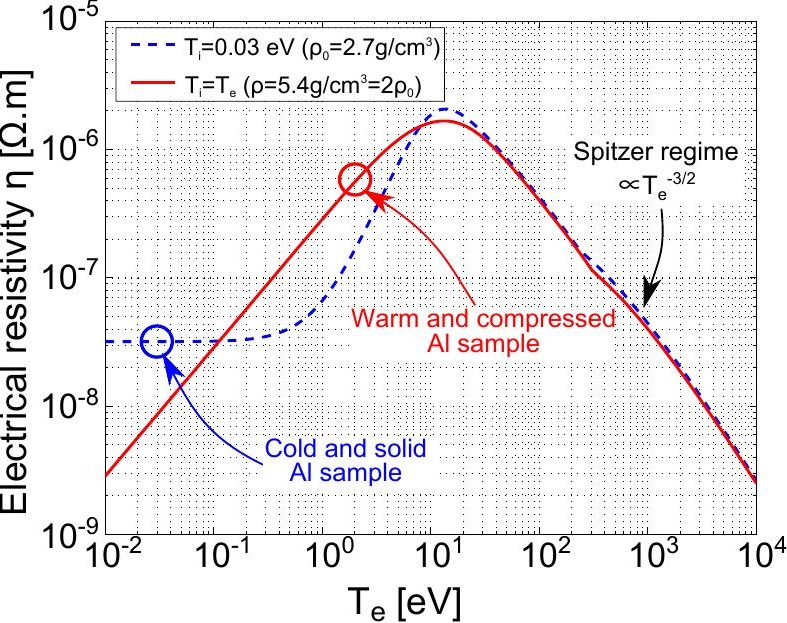




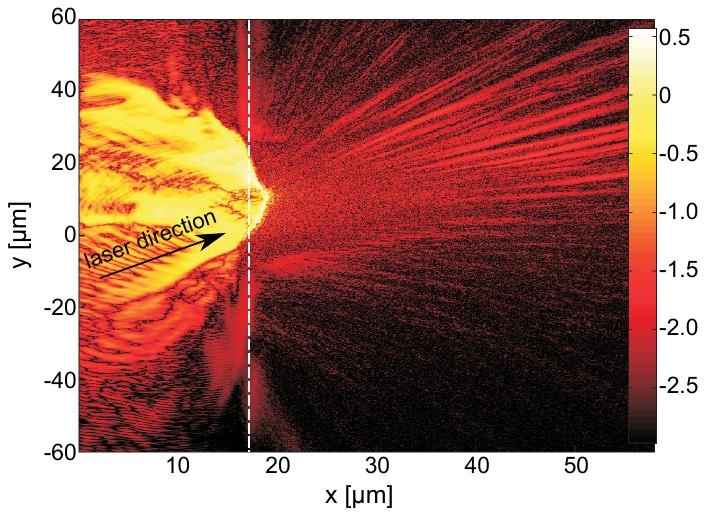




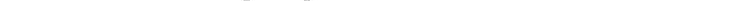


Solid

$10 \mu \mathrm{m}$

$\bullet$

$50 \mu \mathrm{m}$

$40 \mu \mathrm{m}$
Compressed

$6.3 \mu \mathrm{m}(10 \mu \mathrm{m})$

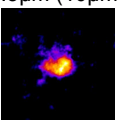

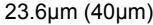

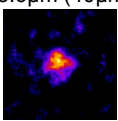

140

\section{.}

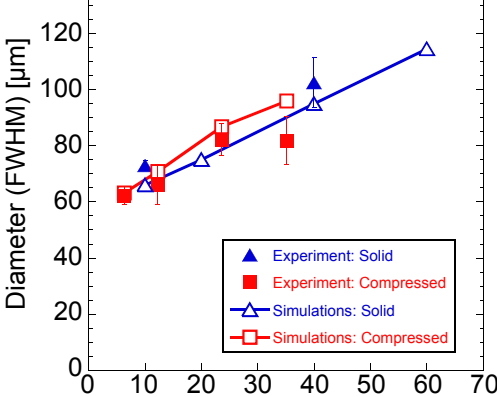
Effective thickness of the Al sample $[\mu \mathrm{m}]$ 
$\left.\begin{array}{l}-\Delta-\text { Solid } \\ \rightarrow \square-\text { Compressed }\end{array}\right\}$ Simulations

$\Delta$ Solid $\}$ Experiment

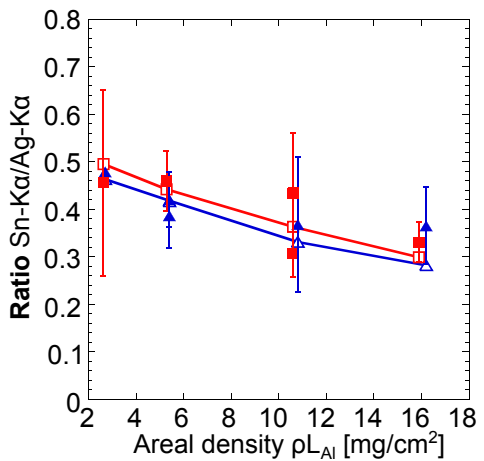




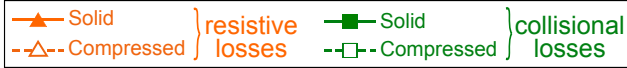

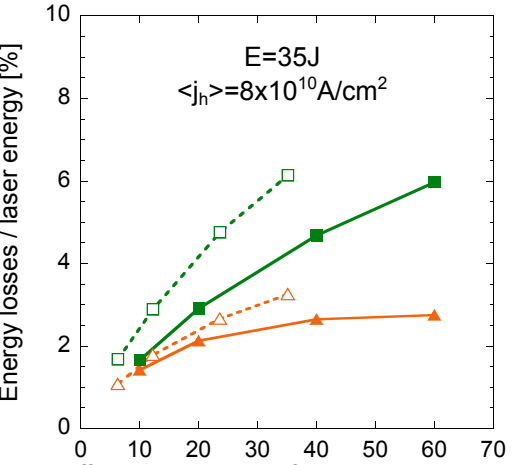

Effective thickness of the Al sample $[\mu \mathrm{m}]$

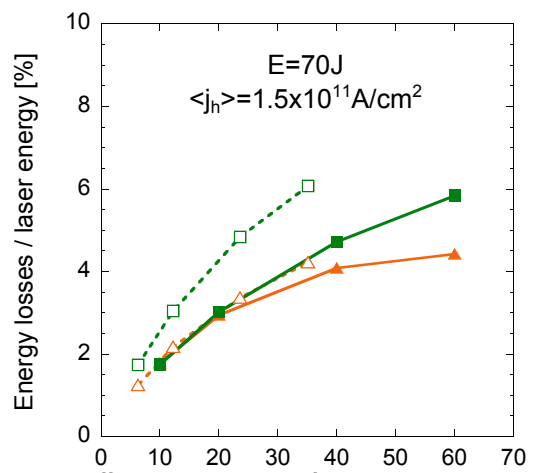

Effective thickness of the Al sample $[\mu \mathrm{m}]$

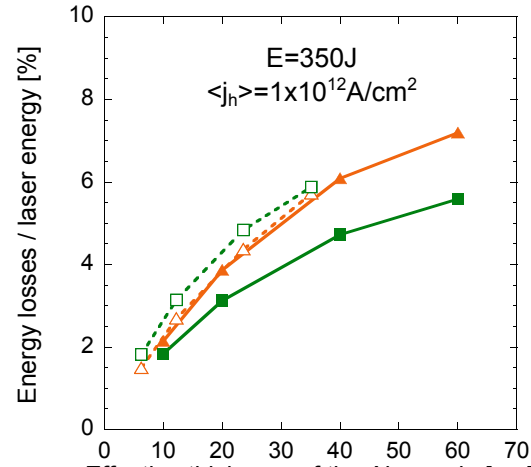

Effective thickness of the Al sample $[\mu \mathrm{m}]$ 


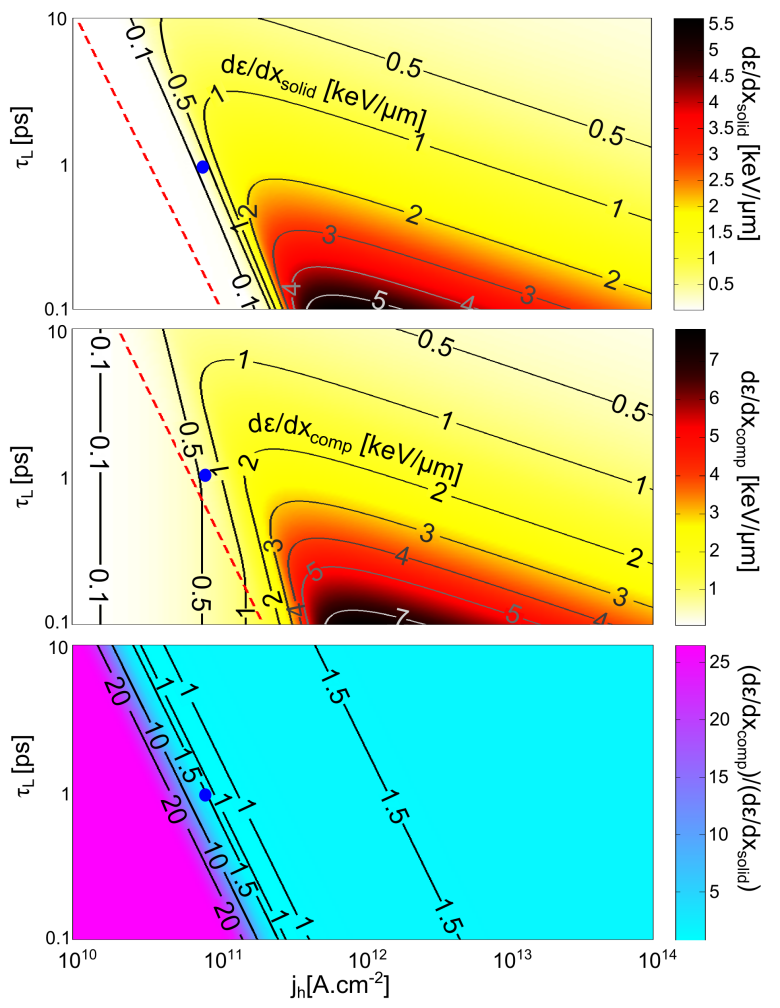

